\title{
Análise de Gamificação em Redes Sociais Gamificadas
}

\section{Rafael Arnold}

Mestrando do Programa de Pós-graduação em Diversidade Cultural e Inclusão Social, Curso de Ciências da computação, Universidade Feevale, Campus II CEP 93525-075 - Novo Hamburgo - RS - Brasil. rafael.arnold@gmail.com

Recibido: 15 Ago 2016 - Revisado: 30 Sep 2016 Aceptado: 30 Oct 2016 - Publicado: 30 Dic 2016

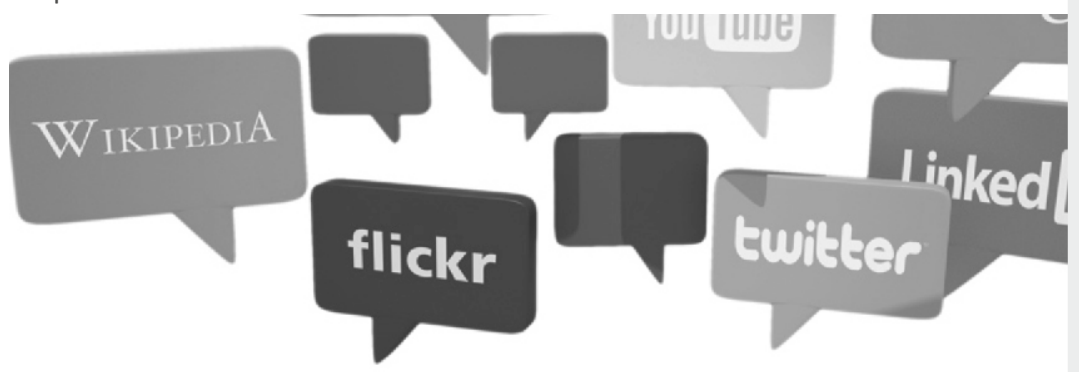

\section{Débora Nice Ferrari Barbosa}

Professora do Programa de Pós-graduação em Diversidade Cultural e Inclusão Social, Mestrado Profissional em Letras, Universidade Feevale, Campus II - CEP 93525-075 . deboranice@feevale.br

\section{Paulo Ricardo Dos Santos}

Acadêmico do curso de Letras - Português e Inglês e bolsista de Iniciação Científica, Curso de Letras Universidade Feevale, Campus I-CEP 93510-250Novo Hamburgo - RS - Brasil. paulords1994@gmail.com

\section{Me. João Mossmann}

Doutorando em Informática na Educação, Curso de Ciências da Computação, Universidade Feevale, Campus II - CEP 93525-075Novo Hamburgo - RS - Brasil.

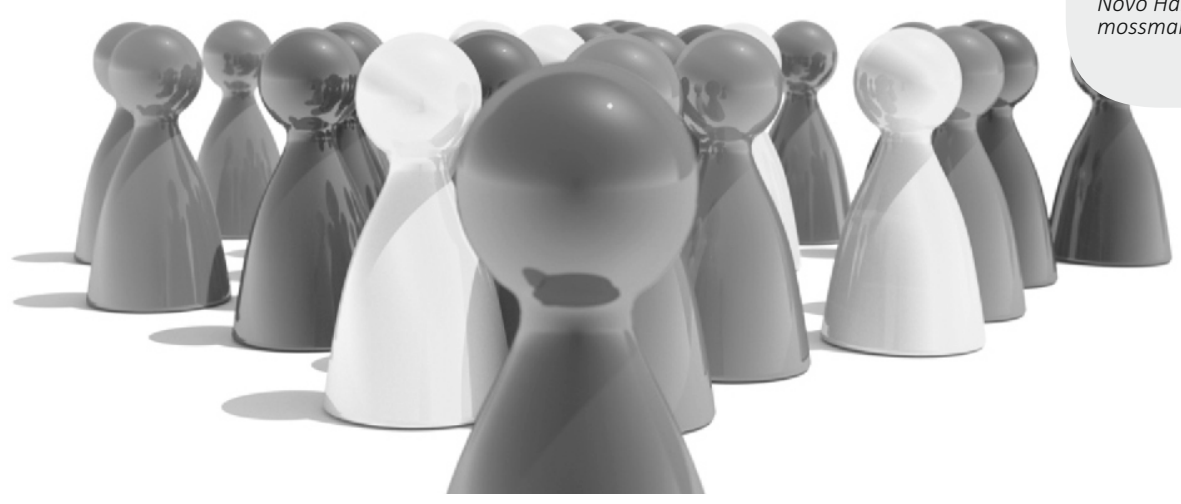

Resumo: O objetivo deste trabalho é avaliar os elementos de gamificação a partir de um modelo de análise de gamificação aplicado a redes sociais gamificadas. Após a análise e compilação dos elementos descritos no modelo, tratou-se de organizar a análise a partir do método utilizado, resultando em cinco categorias. Foram analisados os elementos de gamificação de duas redes sociais educativas e comparadas à Rede Teia, utilizada na AMO em atividades de reforço escolar com crianças e adolescentes que estão em situação de vulnerabilidade social devido ao tratamento oncológico. Pôde-se observar, a partir da análise, a necessidade de aplicar mais elementos de gamificação à Teia para atingir os objetivos de socializar e construir o conhecimento e promover a interação entre os seus usuários na resolução dos problemas apresentados pelos jogos conectados.

Palavras Chave: gamificação; redes sociais; educação.

Abstract: The objective of this study is to evaluate the gamification elements with a gamification analysis model applied to gamificadas social networks. After the analysis and compilation of the elements described in the model, it was organized the analysis from the method used, resulting in five categories. The gamification elements of two educational social networks were analyzed and compared to Rede Teia, which is used in AMO to the reinforcement activities with children and adolescents who are socially vulnerable due to cancer treatment. It can be seen, from the analysis, the need to apply more elements of gamification into the Teia to achieve the objective of socializing and construction of knowledge and to promote the interaction among its users in the solving of the problems presented by online games.

Key words: gamification; social networks; education. 


\section{INTRODUÇÃO}

A gamificação, um fenômeno emergente, estabelece as mecânicas dos jogos em cenários de não-jogos, criando espaços, físicos ou virtuais, mediados pelo engajamento dos indivíduos, na resolução de problemas e na intensificação da aprendizagem. Especificamente, o presente artigo trata de analisar as técnicas de gamificação presentes nas redes sociais educacionais Edmodo (www.edmodo.com) e Passei Direto (www.passeidireto.com), relacionando os possíveis elementos de gamificação a serem implementados na Rede Educacional Teia (http://games.feevale.br/teia). A rede Teia é utilizada na Associação de Assistência em Oncopediatria- AMO, que atende crianças e adolescentes em situação de vulnerabilidade social devido ao tratamento oncológico. A AMO oferece oficinas de informática e atividades de reforço escolar para esses sujeitos que possuem dificuldades de acompanhar a escola durante o período de tratamento.

Segundo Zichermann e Cunningham (2011), o envolvimento de qualquer sujeito deve estar baseado em estruturas de recompensa, reforço e feedbacks, suportadas por mecânicas e sistemáticas que potencializam o envolvimento do indivíduo. A gamificação, que compreende a utilização de técnicas de jogos em atividades de não jogos, pode ser vista como uma forma implícita de motivação. A gamificação e suas técnicas tem sido utilizada há muito tempo, por exemplo, na educação, onde os sujeitos podiam ter suas atividades reconhecidas com estrelinhas, vistas como recompensas, tal como ocorre nos jogos, da mesma forma que, no decorrer do ditado coordenado pela professora, as palavras ficavam cada vez mais complexas ao serem soletradas. Acredita-se que as técnicas de gamificação funcionam como um instrumento motivacional do sujeito, contribuindo para o engajamento deste nas mais variadas atividades propostas. Em grande parte das comunidades online, segundo Nielsen (2006), em torno de $90 \%$ dos usuários não participam ativamente, sendo apenas leitores, 9\% contribuem um pouco e apenas $1 \%$ contribuem com conteúdo. A partir das considerações de Nielsen (2006) quanto à colaboração dos sujeitos nas redes sociais, especificamente as redes sociais educacionais, essas redes utilizam técnicas de gamificação para 0 engajamento dos sujeitos.

Em resposta à questão proposta, o artigo tem como objetivo analisar e comparar as técnicas de gamificação presentes em sistemas gamificados e a forma como esses elementos são aplicados nesses sistemas, especificamente nas redes sociais educacionais Edmodo, Passeidireto e Teia.

A partir de uma revisão bibliográfica, propõe-se um modelo oriundo da análise de duas redes sociais de fins educacionais, com o objetivo de identificar e analisar se os elementos de gamificação estão presentes nas redes e confrontar estes elementos com os presentes na rede Teia. Avellar et al. (2012), a partir de dois documentos que especificam elementos de gamificação necessários em sistemas gamificados, Winning with Gamification (Bunchball, 2011) e SCVNGR playdeck (SCVNGR, 2011), propuseram um modelo teórico para avaliação das redes sociais categorizado em cinco grupos: agendamento de recompensa, reforço, social, dinâmica de progressão e tempo.

Trata-se de uma pesquisa de natureza aplicada, com abordagem qualitativa e de caráter exploratório, pois faz interface entre redes sociais educativas e técnicas de gamificação, oriundas dos jogos digitais. Os dados coletados advém das pesquisas bibliográfica e documental, frente às redes selecionadas para análise. 


\section{FUNDAMENTAÇÃO TEÓRICA}

As redes sociais na internet surgem a partir dos sites de redes sociais, caracterizados pelo cadastro e construção de um perfil e através das relações das conexões entre os diversos perfis. Segundo Recuero (2009), uma rede social pode ser definida como "um conjunto de dois elementos: atores (pessoas, instituições ou grupos; os nós da rede) e suas conexões (interações ou laços sociais)". (Recuero, 2009, p. 24).

Ellison e Boyd (2013) definem sites de redes sociais como uma plataforma de comunicação cujos participantes possuem perfis e com participação de conteúdos produzidos pelo usuário, conteúdos produzidos por outros usuários e dados fornecidos pelos próprios sistemas. Estes, ainda, podem articular suas conexões de forma pública, permitindo a visualização por outros usuários. Esses usuários podem consumir, produzir e interagir com conteúdo gerado por usuários fornecidos por suas conexões na plataforma. Estudos indicam que as redes sociais são utilizadas, em sua maioria, para manter contato entre pessoas, pois as mesmas compartilham fotos e vídeos, entre outros tipos de mensagens, normalmente conforme a confiança atribuída a cada nó da sua rede. Engajar os sujeitos participantes de uma rede social em prol de um objetivo comum está relacionado diretamente com o grau de motivação que este serviço gera. Técnicas de gamificação aplicadas às redes sociais podem fomentar o engajamento desses sujeitos. Zichermann (2011) salienta que a gamificação é a inclusão de mecânicas, estilos, pensamentos e técnicas de design de jogos para envolver os indivíduos na solução de um determinado problema.

A gamificação trabalha para satisfazer alguns desejos dos sujeitos, como: reconhecimento e recompensa, status, realização, competição e colaboração e autoexpressão. As pessoas sentem necessidade por essas coisas, buscando motivação tanto em seu mundo real quanto no virtual.

Ghozland (2010) destaca que a importância da experiência de uma determinada atividade depende de quanto interesse ela pode gerar. Criar e manter o interesse das pessoas é a forma de gerir a sua motivação. Sua motivação é o fator que irá determinar se um indivíduo continuará a realizar determinada atividade depois de algum tempo, assim como se irá atuar na mesma por mais tempo ou finalizá-la. Elementos de gamificação podem influenciar e motivar para a aprendizagem.

Para Cook (2013), a gamificação tem sido apontada como uma nova forma de comunicação social em que os sujeitos interagem e socializam em torno de um conhecimento, com uma estratégia competitiva, divertida e que não deixa de ser agradável. No momento em que o sujeito necessita estabelecer estratégias, métodos e pensar quais são os objetivos de curto, médio e longo prazo para solucionar determinados problemas, o mesmo está exercitando suas habilidades cognitivas, por consequência, uma rede social gamificada pode ser um espaço de aprendizagem. Segundo Alves (2012), um jogo exige que o sujeito possua determinadas estratégias e habilidades cognitivas para solucionar variados problemas.

\section{METODOLOGIA}

Através de uma pesquisa bibliográfica sobre ferramentas e modelos com o objetivo de auxiliar na especificação de elementos de gamificação, encontrou-se no artigo científico de Avellar et al. (2012) um modelo de análise de gamificação aplicado a redes sociais gamificadas. O modelo proposto pelo artigo, com base em Bunchball e Scvngr, foi utilizado como base para avaliação das redes sociais de fins educativos propostas neste artigo. 
Após a análise e compilação dos elementos descritos nos documentos citados acima, tratou-se de estruturar a análise a partir do método utilizado por Avellar et al. (2012), organizando os elementos em grupos que resultaram em cinco categorias: Recompensa: neste grupo foram agrupados os elementos quanto a questão de quando e como recompensas são; Reforço: nessa categoria, são tratadas as questões relacionadas com os tipos de recompensas dadas e oferecidas aos usuários: itens grátis, bens físicos com opção de troca, itens virtuais, pontos, selos; Social: foram agrupadas as questões sobre como os nós das redes interagem entre si, mecânicas virais de jogo, feeds de notícias, equipes; Dinâmica de progressão: agrupa os itens que identificam como o usuário percebe sua progressão no sistema, como níveis e feedback em tempo real; Tempo: categoria relacionada a eventos e ações temporais, como a contagem regressiva, por exemplo. A análise do modelo e das redes sociais de fins educativos foi conduzida pela práxis do pesquisador. A partir de buscas realizadas na internet, foram selecionadas para análise duas redes sociais educacionais, sendo elas:

Edmodo: um ambiente que proporciona uma interação entre escolas, professores e alunos, além da possibilidade do acompanhamento dos pais. As salas de aula podem ser virtualizadas, os professores podem propor atividades e avaliar as mesmas, concedendo recompensas aos seus alunos.

Passeidireto: um ambiente para troca de conhecimento entre alunos, possibilita a postagem de material onde os alunos definem as disciplinas de interesse, trocam mensagens, fazem conexões e são pontuados conforme a participação. Permite ainda a criação de grupos de estudo a partir de suas conexões. A seguir são apresentados os resultados da análise realizada:
Tabela 1. Recompensa.

\begin{tabular}{|l|c|c|}
\hline & Edmodo & Passeidireto \\
\hline $\begin{array}{l}\text { Existe pelo menos um } \\
\text { sistema de problema - } \\
\text { resposta - recompensa? }\end{array}$ & Sim & Sim \\
\hline $\begin{array}{l}\text { Existem recompensas } \\
\text { baseadas em tempo fixo } \\
\text { e/ou variável (por } \\
\text { exemplo, a cada x } \\
\text { minutos o usuário ganha } \\
\text { ponto(s))? }\end{array}$ & Não & Não \\
\hline $\begin{array}{l}\text { Existem recompensas } \\
\text { baseadas em número de } \\
\text { ações fixo e/ou variável } \\
\text { (por exemplo, a cadax } \\
\text { comentários/postagens o } \\
\text { usuário ganha ponto(s))? }\end{array}$ & Sim & \\
\hline
\end{tabular}

Tabela 2. Reforço.

\begin{tabular}{|l|c|c|}
\hline & Edmodo & Passeidireto \\
\hline Existem punições? & Não & Sim \\
\hline $\begin{array}{l}\text { É possível evitar } \\
\text { punições através de } \\
\text { ações? }\end{array}$ & Não & Não \\
\hline $\begin{array}{l}\text { É possivel ganhar } \\
\text { recompensas através } \\
\text { apenas do esforço dos } \\
\text { outros? }\end{array}$ & Não & Não \\
\hline $\begin{array}{l}\text { O usuário recebe } \\
\text { reconhecimento } \\
\text { virtual por suas ações, } \\
\text { através de selos, } \\
\text { troféus ou afins? }\end{array}$ & Sim & Sim \\
\hline
\end{tabular}

Tabela 3. Social.

\begin{tabular}{|l|c|c|}
\hline & Edmodo & Passeidireto \\
\hline $\begin{array}{l}\text { É possivel se } \\
\text { juntar a outros }\end{array}$ & Não & Sim \\
$\begin{array}{l}\text { usuários para } \\
\text { solucionar } \\
\text { problemas? }\end{array}$ & Sim & Sim \\
\hline $\begin{array}{l}\text { Existem } \\
\text { rankings? }\end{array}$ & & \\
\hline
\end{tabular}




\begin{tabular}{|l|l|l|}
\hline $\begin{array}{l}\text { Os rankings são } \\
\text { divididos por } \\
\text { categorias? }\end{array}$ & Sim & Sim \\
\hline $\begin{array}{l}\text { Existem feeds que } \\
\text { mostrem as } \\
\text { ações que os } \\
\text { outros usuários } \\
\text { estão realizando } \\
\text { em tempo real? }\end{array}$ & Não & Não \\
\hline $\begin{array}{l}\text { O usuário pode } \\
\text { presentear outros } \\
\text { usuários de } \\
\text { alguma maneira? }\end{array}$ & Não & Não \\
\hline $\begin{array}{l}\text { O usuário pode } \\
\text { conversar com } \\
\text { outros usuários? }\end{array}$ & Sim & Sim \\
\hline
\end{tabular}

Tabela 4. Dinâmica de progressão.

\begin{tabular}{|l|c|c|}
\hline & Edmodo & Passeidireto \\
\hline $\begin{array}{l}\text { O usuário recebe } \\
\text { informação de } \\
\text { modo gradual? }\end{array}$ & Sim & Não \\
\hline $\begin{array}{l}\text { O usuário pode } \\
\text { ganhar niveis } \\
\text { realizando ações? }\end{array}$ & Sim & Sim \\
\hline $\begin{array}{l}\text { O sistema informa } \\
\text { ao usuário } \\
\text { imediatamente } \\
\text { quando algo } \\
\text { importante } \\
\text { acontece? }\end{array}$ & Sim & Sim \\
\hline $\begin{array}{l}\text { O usuário tem } \\
\text { acesso a listas de } \\
\text { tarefas } \\
\text { enumeradas } \\
\text { individualmente? }\end{array}$ & Sim & Não \\
\hline
\end{tabular}

Tabela 5. Tempo.

\begin{tabular}{|l|c|c|}
\hline & Edmodo & Passeidireto \\
\hline $\begin{array}{l}\text { O usuário se } \\
\text { sente } \\
\text { encorajado a } \\
\text { retornar ao } \\
\text { sistema após } \\
\text { algum tempo? }\end{array}$ & Não & Não \\
\hline $\begin{array}{l}\text { Existem ações } \\
\text { que o usuário } \\
\text { precisa realizar } \\
\text { com } \\
\text { frequência } \\
\text { definida? }\end{array}$ & Sim & Não \\
\hline $\begin{array}{l}\text { Existem } \\
\text { problemas } \\
\text { com limite de } \\
\text { tempo? }\end{array}$ & Sim & Não \\
\hline
\end{tabular}

Tabela 6. Social.

\begin{tabular}{|l|c|c|}
\hline & Edmodo & Passeidireto \\
\hline Recompensa & $2 / 3$ & $2 / 3$ \\
\hline Reforço & $1 / 4$ & $2 / 4$ \\
\hline Social & $3 / 6$ & $4 / 6$ \\
\hline $\begin{array}{l}\text { Dinâmica de } \\
\text { Progressão }\end{array}$ & $4 / 4$ & $2 / 4$ \\
\hline Tempo & $2 / 3$ & $0 / 3$ \\
\hline Total & $\mathbf{1 2 / 2 0}$ & $\mathbf{1 0 / 2 0}$ \\
\hline
\end{tabular}

Os elementos de recompensas, socialização e dinâmica de progressão ficam explícitos em ambas as redes, a partir do cumprimento de tarefas propostas. Edmodo possui tarefas nativas da rede que são disponibilizadas para resolução por parte do aluno. Já a rede Passeidireto, gera recompensas a partir da participação dos sujeitos e a partir da interação com o 
sistema. Os elementos de reforço e tempo dos sistemas analisados são os menos explorados. De maneira geral, ambos os sistemas possuem nativamente os elementos de gamificação que objetivam a motivação de seus usuários.

\section{ANÁLISE E DISCUSSÃO}

Analisando as técnicas de gamificação presentes nas redes sociais educacionais Edmodo e Passeidireto, é possível destacar que ambas possuem grande parte dos elementos necessários em um sistema gamificado. De forma intuitiva, segundo Lee e Hammer (2011), a gamificação tem um grande potencial para motivar os alunos e tornar a escola mais atraente. É dessa forma que o sistema Edmodo busca engajar os seus usuários, trazendo para o seu contexto a sala de aula, professores, alunos e até mesmo pais de alunos. O sistema permite aos professores criarem tarefas, pontuarem e ou graduarem os alunos conforme o atingimento das metas, conceder medalhas, trocar mensagens entre a turma e adicionar conteúdo através de upload. O sistema também disponibiliza tarefas no formato de quiz, concedendo pontuação aos alunos conforme a progressão na atividade, proporcionando uma experiência diferente da vivenciada na sala de aula física.

Simões et al. (2012), destaca que as redes sociais se tornaram mais populares e deram origem a jogos sociais. Seguindo essa linha de análise, a rede Passeidireto caracteriza-se como um ambiente social de apoio educativo e possui elementos de gamificação perceptíveis para incentivar os usuários a compartilharem materiais e a realizarem download dos materiais compartilhados por outros sujeitos. 0 sistema também atribui um ranking ao usuário, à instituição de ensino e ao curso vinculado no momento do cadastro e o sujeito recebe um status, que muda conforme a pontuação atingida a partir da interação com o sistema. Além disso, permite conexão com outros usuários e também a troca de mensagens entre eles. Assim, a rede Passeidireto deixa muito evidente a utilização das técnicas de gamificação para engajamento do usuário. Ambas as redes possuem valorosos elementos de um sistema gamificado, elementos que atraem diferentes tipos de usuários de acordo com o público alvo de cada rede social. Ao aplicarem elementos que motivem usuários distintos, todos terão algum tipo de motivação, atraindo diferentes públicos para as redes.

Dado o complexo sistema de aprendizagem e a necessidade de melhorias do sistema de ensinoaprendizagem para um aumento do engajamento dos sujeitos, não podemos, segundo Alves e Santos (2006), ignorar os espaços das tecnologias da informação e comunicação (TICS) no processo de aprendizagem dos indivíduos. Ainda, as TICs possibilitam novos espaços e incentivam os sujeitos a serem capazes de autocriarem-se e darem sentido à existência do coletivo, potencializando a prática coletiva das redes de relações. (Alves e Santos, 2006).

\section{CONCLUSÃO}

Sendo a rede Teia uma rede social educativa com o objetivo de auxiliar no processo de ensinoaprendizagem de crianças e adolescentes em situação de vulnerabilidade social devido ao tratamento oncológico, a Teia é integrada a um conjunto de jogos para dispositivos móveis e deve proporcionar estratégias pedagógicas motivadoras e significativas para os usuários através do uso de técnicas de gamificação envolvendo os jogos e a rede.

O estudo das redes Edmodo e Passeidireto contribuiu para um melhor entendimento dos elementos de gamificação a serem aplicados na Rede Teia. Atualmente os elementos de gamificação presentes na rede Teia são aplicados de forma incremental, o ranking é feito através de um sistema de pontos, atribuindo níveis para os usuários. Além disso, o 
jogador pode se comparar a outro jogador da sua rede de amigos e perceber sua evolução.

A Rede Teia possui um diferencial, apesar de não ser um jogo, por fazer a interação com jogos digitais educativos, sendo isso uma das partes do sistema, pois os jogos que são jogados através de dispositivos móveis são a outra parte do sistema. Os dados a respeito dos jogos e jogadores estão distribuídos, tanto no próprio jogo, quanto na rede.

A Rede Teia tem os objetivos de socializar o conhecimento e promover a interação entre os seus usuários através da construção do conhecimento para a resolução dos problemas apresentados pelos jogos conectados à rede. Segundo Gee (2007) os bons games incorporam princípios de ensinoaprendizagem na sua própria concepção e exigem dos jogadores o domínio de novas práticas de letramento que estão mais relacionadas com práticas sociais do que individuais. Acredita-se que, através de práticas sociais de aprendizagem, da troca de conhecimento na Teia entre os seus usuários e com a ajuda de técnicas de gamificação para o engajamento dos sujeitos, o objetivo proposto pela rede seja atendido. Este estudo auxiliou na identificação das necessidades específicas de gamificação em que a Teia necessita de melhorias. Dessa forma, a partir da análise realizada, serão estudadas técnicas especificas de gamificação que possam ser incrementadas a Teia. Entendemos que a Teia estará apta ao ensino gamificado quando a combinação de elementos como desafios, metas, objetivos, conquistas e técnicas dos games não despertem apenas o interesse no aluno do jogar pelo jogo, mas sim, do jogar pela motivação do ensinoaprendizado.

Agradecimentos Os autores agradecem ao Conselho Nacional de Desenvolvimento Científico e Tecnológico - CNPq e à Universidade Feevale pelo apoio à pesquisa

\section{REFERÊNCIAS}

Alves, L. Agência da Ufba: Lynn Alves fala sobre games, educação e Parque Tecnológico. Disponível em http://www.uneb.br/2012/05/05/agencia-da-ufbalynn-alves-fala-sobre-games-educacao-e-parquetecnologico-teste/. Acesso em 30 mai 2016.

Bunchball. Winning with gamification: tips from the expert's playbook. San Jose, EUA. Disponível em http://www.bunchball.com/playbook/pbbbtombston e.shtml Acesso em: 30 mai 2016.

Cook, W. (2013). Five Reasons Why You Can't Ignore GAMIFICATION. Disponível em http://m.cedmaeurope.org/newsletter\%20articles/Clomedia/Five\%2 Oreasons\%20you\%20Cannot\%20Ignore\%20Gamificat ion\%20(May\%2013).pdf. Acesso em: 30 mai 2016.

Nicole. B. Ellison; D. Boyd. (2013). Sociality through Social Network Sites. In: The Oxford Handbook of Internet Studies. In Oxford Universitário Pressão. 151172 Disponível e m http://www.danah.org/papers/2013/SocialityThruSN S-preprint.pdf. Acesso em 13 jun 2016.

Gee, J. (2007). What video games have to teach us about learning and literacy. (2a. edição). Palgrave Macmillan.

Ghozland, D. (2011). Designing for Motivation, Gamasutra, pp.1-9. Disponível em http://www.gamasutra.com/view/feature/1419/desi gning_for_motivation.php. Acesso em 30 mai 2016.

Lee, J. and Hammer, J. (2011). Gamification in education: What, how, why bother? Disponível em https://www.academia.edu/570970/Gamification_in Education_What_How_Why_Bother. Acesso em 01 jun 2016. 
Nielsen, J. (2006). Participation Inequality: Encouraging More Users to Contribute. (2006) Disponível em

https://www.nngroup.com/articles/participationinequality. Acesso em 01 jun 2016.

Recuero, R. (2009). Redes Sociais na Internet. Porto Alegre: Sulina.

Recuero, R., Bastos, M. and Zago, G. (2015). Análise de Redes para Mídia Social. (1.Ed). Porto Alegre.

Santos, E. and Alvez, L. (2016). Práticas Pedagógicas e Tecnologias Digitais. Disponível em https://books.google.com.br/books?id=yL9mOXySqL g $C \& \mid p g=P A 3 \& o t s=A$ e 7 h $s t 1$ $17 \& d q=\mid$ y $n n \% 20$ a $\mid$ ve $s \&|r \& h|=p t-$ $B R \& p g=P A 28 \# v=$ onepage $\& q \& f=$ false. Acessado em 31 mai 2016.
Simões, R., Redondo, D., Vilas, A. (2012). A social gamification framework for a K-6 learning platform. In Elseviers Journals - Computers in Human Behavior. Disponível em

https://www.researchgate.net/publication/2308548 06_A_social_gamification_framework_for_a_K6_learning_platform. Acesso em 30 mai 2016.

Scvngr. SCVNGR playdeck, (2011). EUA. Disponível em: http://techcrunch.com/2010/08/25/scvngrgamemechanics Acesso em 30 mai 2016.

Zichermann, G. and Cunningham, C. (2011). Gamification by Design: Implementing Game Mechanics in Web and Mobile Apps. Sebastopol, CA: O'Reilly Media, Inc.. 